\title{
JÉRÔME BURIDANT
}

\section{Croissance industrielle et demande énergétique Le cas du bois $\left(\mathrm{XVIII}^{\mathrm{e}}-\mathrm{XIX}^{\mathrm{e}}\right.$ siècles $)$}

L'historiographie française a longtemps considéré la révolution industrielle comme une rupture majeure dans l'histoire économique et sociale. Cette notion a été forgée en 1837 par Adolphe Blanqui dans son »Histoire de l'économie politique en Europe«. Comparant les destinées de la France et de la Grande-Bretagne, celui-ci remarquait des divergences de parcours:

tandis que la Révolution française faisait ses grandes expériences sociales sur un volcan, l'Angleterre commençait les siennes sur le terrain de l'industrie. La fin du XVIII ${ }^{\mathrm{e}}$ siècle y était signalée par des découvertes admirables, destinées à changer la face du monde et à accroître de manière inespérée la puissance de leurs inventeurs [...]. À peine éclose du cerveau de ces deux hommes de génie, Watt et Arkwright, la révolution industrielle se mit en possession de l'Angleterre.

Adoptée par la communauté des historiens et des économistes, cette notion allait rapidement servir à caractériser les mutations enregistrées au XIX ${ }^{\mathrm{e}}$ siècle dans la plupart des pays occidentaux: accélération de la croissance économique, qui passe d'environ $0,5 \%$ à $2 \%$ par an, développement des industries textile et sidérurgique, progrès des transports terrestres et maritimes, passage à de nouvelles énergies comme le charbon puis l'électricité1. En privilégiant les ruptures techniques sur les dynamiques de longue durée, les historiens ont longtemps été conduits à favoriser le rôle de l'innovation dans la croissance économique ${ }^{2}$. Cette focalisation sur les phénomènes de rupture permettait d'abord d'intégrer les débats et les théories économiques, comme celles de Walt Whitman Rostow ou de Joseph Aloïs Schumpeter ${ }^{3}$. Elle présentait aussi l'avantage de conforter les césures de l'enseignement de l'histoire, traditionnelle en France, séparant de manière tranchée l'histoire moderne $\left(\mathrm{XVI}^{\mathrm{e}}-\mathrm{XVIII}{ }^{\mathrm{e}}\right.$ siècles) de l'histoire contemporaine $\left(\mathrm{XIX}^{\mathrm{e}}-\mathrm{XXI}^{\mathrm{e}}\right.$ siècles): aux modernistes l'histoire d'un passé révolu, celui de l'Ancien Régime, aux contemporanéistes l'histoire des ruptures conduisant au monde d'aujourd'hui. Dans ces conditions, les interrogations portaient plus sur l'articulation entre les évolutions démographiques, agricoles et industrielles, que sur les mutations énergétiques, la substitution du charbon au bois semblant évidente au moment où

1 J. Buridant, A. FigliUZZI, G. NoËL et al., Histoire des faits économiques, Paris 2001, p. 53-54.

2 Voir par exemple Jean-Pierre RIOUX, La révolution industrielle, 1780-1880, Paris 1971; Patrick VERLEY, La Révolution industrielle, Paris 1997.

3 Walt Whitman Rostow, Les étapes de la croissance économique. Un manifeste non-communiste, Paris 1960, rééd. Paris 1963; Joseph Aloïs SCHUMPETER, Théorie de l'évolution économique, Paris 1925. 
l'innovation technique est servie par la découverte de ressources abondantes ${ }^{4}$. En dépit de son importance, la question des mutations énergétiques reste relativement neuve. Elle a d'abord été posée avec force par des historiens de l'industrie comme Denis Woronoff, pour déboucher sur de multiples enquêtes, tant du côté de la ressource en charbon de terre que sur celle de la ressource en bois ${ }^{5}$. Le fait que l'industrie ait basé sa croissance sur la houille ne va pas de soi, et constitue à bien des égards une image réductrice des phénomènes. Techniquement, le bois constituait une énergie parfaitement maîtrisée qui pouvait fournir des températures supérieures à celles du charbon, et des chauffes de meilleure qualité. Si les rythmes de passage à la chauffe au charbon s'expliquent naturellement par des innovations techniques, elles sont motivées à la base par les coûts relatifs des différents combustibles, et par la menace d'une pénurie de bois, toujours plus inquiétante avec le temps.

\section{CHRONIQUE D’UNE CRISE ANNONCÉE}

\section{UN PROBLÈME D'AMPLEUR NATIONALE}

$\mathrm{Au}$ début du règne de Louis XIV, le contrôleur général des Finances Jean-Baptiste Colbert avait lancé un vaste programme de réforme des forêts françaises, visant à restaurer les peuplements, limiter les abus des riverains, réorganiser les coupes, pour fournir à la Marine, à l'industrie comme à la consommation urbaine des ressources abondantes et régulières. La réformation des forêts royales (1661) et l'ordonnance de Saint-Germain en Laye (août 1669) devaient conduire à une stabilisation des surfaces, à une normalisation de la production et à une régularisation de l'offre, évitant à jamais les crises d'approvisionnement rencontrées dans les années 1540-1550, ou 1650 $1670^{6}$. En dépit de tous ces efforts, on remarque au XVIII ${ }^{\mathrm{e}}$ siècle une persistance, voire une aggravation des difficultés, notamment en ville où l'approvisionnement en combustible n'est plus garanti lors d'hivers rigoureux, notamment en 1714-1715 ou en 1740-1742. Après 1770, on constate une brutale envolée des prix qui montre que la crise tend désormais à devenir structurelle.

Dans les années 1930, Ernest Labrousse avait tenté d'étudier l'évolution des prix des bois pour l'intégrer à son »Esquisse du mouvement des prix et des revenus en France «7. S'intéressant exclusivement aux prix à la consommation, celui-ci constatait qu'ils avaient augmenté de plus de $91 \%$ entre 1726 et 1789 , correspondant à la hausse

4 Voir par exemple l'analyse du Belge Paul BAIROCH, Le tiers monde dans l'impasse: le démarrage économique du XVIII ${ }^{\mathrm{e}}$ au XX $\mathrm{XX}^{\mathrm{e}}$ siècle, Paris 1971.

5 Denis WorOnOFF, L'industrie sidérurgique en France pendant la Révolution et l'Empire, Paris 1984; ID., Histoire de l'industrie en France: du XVI' siècle à nos jours, Paris 1994; Nadège SoUGY, Les charbons de la Machine: valorisation et commercialisation des produits d'une houillère nivernaise, de 1838 à 1938, université de Paris I - Panthéon-Sorbonne: thèse de $3^{\mathrm{e}}$ cycle, 2001.

6 Michel DEvĖze, Une admirable réforme administrative: la grande réformation des forêts royales sous Colbert (1661-1680), Nancy 1962.

7 Ernest LABROUSSE, Esquisse du mouvement des prix et des revenus en France au XVIII ${ }^{\mathrm{e}}$ siècle, t. II: Les prix (fin), les revenus, Paris 1933, rééd. Paris 1984, p. 343-348. 
la plus forte par rapport à celle de tous les autres produits qu'il avait pu jusqu'alors observer. Si ce constat était exact, la démarche adoptée n'était toutefois pas exempte de critiques. L'historien était parti des états de prix de quatre généralités périphériques (Montauban, Riom, Grenoble, Besançon et Strasbourg), en excluant le Bassin parisien où les variations étaient les plus fortes. Faute de sources directement accessibles, il s'était aussi limité aux prix des bois livrés au consommateur, prix qui incorporait des données complexes comme le prix du bois sur pied, la coupe, le transport par voie d'eau et voie de terre, ainsi que les bénéfices des marchands.

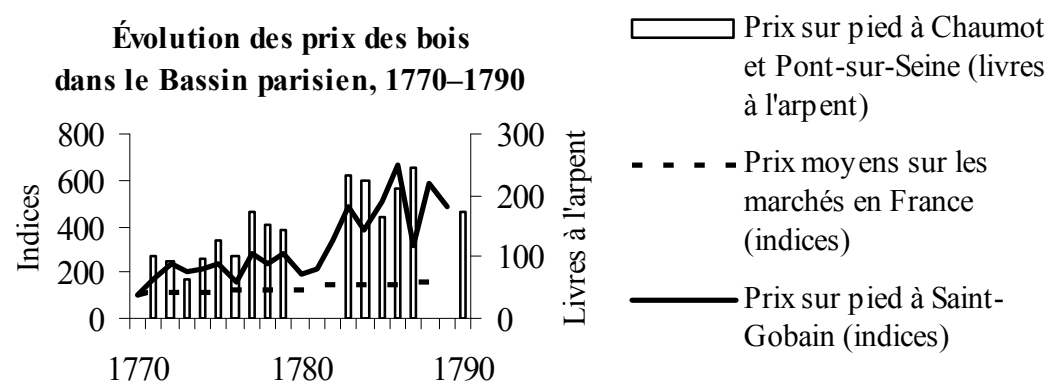

Pour mieux comprendre l'envolée des prix, il faut donc d'abord mesurer l'évolution des prix des bois sur pied en dépouillant les adjudications de bois, lorsqu'elles sont continues et homogènes. Dans le Bassin parisien, les prix sont multipliés par trois en vingt ans, parfois même davantage dans certains bassins d'approvisionnement industriels ${ }^{8}$. Dans ces deux terribles décennies, les accidents conjoncturels paraissent naturellement plus difficiles encore, comme en 1783-1784 (hiver très froid et crue de débâcle) ou en 1792-1793. Ces événements conduisent à une véritable panique dans tout le pays.

La première réponse à cette crise est le lancement de grandes enquêtes administratives, qui visent à faire un premier point sur la ressource ligneuse, pour apporter des réformes dans les bois les plus dégradés, rechercher les zones restant éventuellement encore excédentaires, et mieux mettre en adéquation la demande et l'offre. La première enquête générale est lancée en 1771 par la Table de marbre de Paris ${ }^{9}$. Cette institution dont le ressort s'étend au-delà de celui du Parlement de Paris, sur plus d'un tiers du royaume, constitue le niveau supérieur de l'administration des Eaux et Forêts. Elle doit

8 Pour le graphique, les prix à Chaumot et à Pont-sur-Seine sont tirés de: Patricia COLFORT, Le domaine de Chaumot à la veille de la Révolution française (1771-1793), université de Reims Champagne-Ardenne: mémoire de master II, 2006; Aurélie LoUIS, Le domaine de Pont-surSeine au temps de Xavier de Saxe (1775-1790), mémoire de maîtrise, université de Besançon 2004, et Arch. dép. Aube E* 2807-2808; les prix en France de LABROUSSE, Esquisse du mouvement des prix (voir n. 7), p. 343-348, les prix à Saint-Gobain de Jérôme BURIDANT, Espaces forestiers et industrie verrière $\left(\mathrm{XVII}^{\mathrm{e}}-\mathrm{XIX}^{\mathrm{e}}\right.$ siècle), Paris 2005, p. 207-227.

9 BNF, ms. 7867. 
son nom à une véritable table de marbre, placée dans la grande salle du palais et détruite par un incendie en 1618. Cette enquête porte sur les bois des gens dits de »mainmorte«, c'est-à-dire sur les communautés ecclésiastiques et d'habitants. Elle vise à connaître la localisation, le prix et le débit des bois, mais aussi à mesurer la régularité des aménagements. Elle démontre surtout que les maîtrises des Eaux et Forêts n'ont qu'une connaissance très partielle des forêts qu'ils ont sous tutelle, qu'une grande partie des bois des communautés ne sont ni bornés, ni aménagés, et que l'œuvre de réformation entreprise au XVII ${ }^{\mathrm{e}}$ siècle est loin d'être terminée. La seconde enquête d'envergure est lancée par l'intendant de finances Bonnaire des Forges, lors de la disette de bois de 1783-178410. S'appuyant sur le réseau des intendants et des subdélégués en province, cette enquête est adressée aux maires et syndics de toutes les communautés d'habitants pour connaître les superficies en bois, les prix du combustible, le débit des bois et les possibilités d'exportation, mais surtout l'ampleur de la consommation locale, le nombre de forges, fourneaux, fours à chaux, verreries, etc. Mieux que la précédente, elle vise à faire un lien plus étroit entre les lieux de production et les zones de consommation. Elle se double au même moment d'enquêtes plus ponctuelles menées par le Bureau de la Ville pour l'approvisionnement de la capitale, par le truchement de ses propres subdélégués. D'une certaine manière, ces investigations rentrent dans un grand mouvement de doléances contre l'industrie, en rendant implicitement les établissements industriels responsables de la pénurie.

Dans un contexte de crise, l'heure est toujours à la recherche de responsables. Les premiers à subir les critiques sont souvent les industriels ${ }^{11}$. Il est vrai qu'au $\mathrm{XVIII}{ }^{\mathrm{e}}$ siècle, les phénomènes de concentration et la croissance des capacités de production ont donné naissance à de très gros établissements, qui sont autant de »gouffres dévorants « en combustible ligneux. À cette époque, un haut fourneau consomme environ 15000 stères de bois par an, principalement en charbonnette, c'est-à-dire en bois de petit calibre destiné à la confection du charbon. Une verrerie de »menu-verre«, qui produit des bouteilles et de la gobeleterie par soufflage, réclame quant à elle 10000 à 15000 stères en billette, bois de plus gros calibre débité par refente. Deux autres établissements les dépassent encore plus largement. La compagnie de Saint-Gobain, qui produit des grandes glaces par coulage, tout comme la verrerie de Saint-Louis en Lorraine, consomment chacune près de 30000 stères par an. À l'échelle du Bassin parisien, la concurrence entre la demande industrielle et la demande urbaine donne naissance à des clivages spatiaux particulièrement nets ${ }^{12}$. Autour de Paris se développe d'abord une auréole d'approvisionnement en bois neuf par voie de terre, relayée par une aire d'approvisionnement en charbon de bois, quoique relativement limitée dans la partie nord-est de l'Île-de-France (forêt d'Halatte par exemple). Ce charbon, encore

10 Déposée dans les archives départementales en série C.

11 Voir Denis WoronofF, La politique des autorisations d'usines et la question du bois, dans: ID. (dir.), Forges et forêts: recherches sur la consommation proto-industrielle de bois, Paris 1990, p. $57-86$.

12 Jérôme BuRIDANT, Transport des bois et logiques spatiales au XVIII ${ }^{\mathrm{e}}$ siècle, dans: Andrée CORVOL (dir.), Forêt et transports traditionnels, Paris 2004 (Cahier d'études forêt, environnement et société, $\mathrm{XVI}^{\mathrm{e}}-\mathrm{XX}^{\mathrm{e}}$ siècle, 14), p. 95-102. 
voituré par charrois, supporte mieux les frais de transport que la bûche, car il incorpore davantage de valeur ajoutée. Au-delà d'une soixantaine de kilomètres commence l'aire du flottage, irriguée par les bois et les forêts de Compiègne, Laigue ou VillersCotterêts, sur l'Oise, la Marne, la Seine et l'Yonne. Ces bois, de qualité moindre en raison de leur transit dans l'eau, restent très concurrentiels à cause de la modicité des frais de transport. Au-delà, le clivage s'exprime longtemps par un effondrement des prix. Le plus souvent est alors adoptée une production à plus haute valeur ajoutée. Il peut s'agir d'une orientation vers une sylviculture de qualité, pour la fourniture du merrain à tonneaux ou du bois d'œuvre, en privilégiant la futaie sur le taillis. Mais le choix entraîne plus souvent une valorisation énergétique locale destinée à l'alimentation des forges, verreries et autres fourneaux. L'absence, réelle ou supposée, de débouchés pour les bois constitue d'ailleurs longtemps le premier argument avancé pour obtenir l'autorisation d'implantation d'un établissement industriel. Mais cette logique trouve désormais ses limites dans l'élargissement de l'aire d'attraction de la capitale, liée à l'aménagement des cours d'eau entrepris pour répondre à la »disette« croissante de bois. La progression de la flottabilité des cours d'eau induit de fait un surenchérissement du combustible en amont, qui nuit aux intérêts des industriels, refusant cette concurrence. Dans la seconde moitié du XVIII ${ }^{\mathrm{e}}$ siècle, les zones de contact entre les aires d'approvisionnement urbain et les aires d'approvisionnement industriel deviennent des zones de conflits, qui témoignent de l'âpreté des tensions pour la maîtrise de la ressource énergétique.

Ce qui remonte de toutes ces enquêtes est beaucoup plus complexe que ce que l'on pouvait en attendre. Si l'industrie est régulièrement critiquée, elle n'est pas systématiquement stigmatisée. Les fautifs sont nombreux: les seigneurs et leur passion immodérée pour la chasse, la prolifération du gibier causant des dommages irrémédiables aux peuplements forestiers; les habitants eux-mêmes qui dégradent à l'envi les bois usagers faute de surveillance; les officiers des Eaux et Forêts, qui cherchent surtout à rentabiliser leur office et manquent cruellement de formation technique... Avant même les cahiers de doléances, ces enquêtes de terrain libèrent une parole politique qui débouche sur de multiples contestations.

Du côté de la demande, les causes les plus profondes de la crise sont souvent négligées, même si elles sont aujourd'hui plus faciles à déterminer. La hausse de la demande résulte d'abord et avant tout par la croissance accélérée de la population. De 1715 à 1789 , le royaume passe de 21 à 28-29 millions d'âmes, réalité dont les contemporains n'ont pas conscience avant les travaux de l'abbé d'Expilly'13. Cette hausse globale cache une croissance urbaine encore plus soutenue, notamment à Paris. En un peu plus de trois siècles, la population de la capitale est en effet multipliée par quinze, passant d'environ 150000 habitants vers 1650 , à 510000 à la fin du règne de Louis XIV (vers 1715), à 604000 vers 1780, et à 2,3 millions un siècle plus tard. Plus en retrait, d'autres villes comme Versailles, Troyes, Auxerre ou Rouen enregistrent des rythmes de croissance similaires, sinon encore plus vifs, qui préfigurent ceux de la

13 Jean-Joseph D'EXPILLY, De la population de la France, Paris, Amsterdam 1765; ID., Tableau de la population de la France, s.l.n.e. 1780. 
seconde moitié du XIX ${ }^{\mathrm{e}}$ siècle. Dans le monde urbain, on note aussi parallèlement une forte augmentation des besoins individuels. À la fin du XVI ${ }^{\mathrm{e}}$ siècle, les Parisiens consomment environ un stère par an et par personne: le bois sert à chauffer les aliments et à atténuer les rigueurs des plus grands hivers, tout au plus. À la veille de la Révolution, ils consomment désormais le double, soit plus d'une tonne par personne. Cette croissance des besoins s'explique surtout par l'évolution des mentalités, le chauffage se diffusant progressivement dans toutes les pièces de la maison. Elle est un peu atténuée par des progrès dans les techniques de chauffage comme les poêles, les cheminées à conduits multiples, les réflecteurs placés devant le foyer, les fourneaux remplaçant l'âtre. La demande en combustible ligneux suit donc une croissance exponentielle jusqu'au passage au charbon de terre, dans la seconde moitié du XIX ${ }^{\mathrm{e}}$ siècle. Elle passerait de 315000 stères dans la seconde moitié du XVI ${ }^{\mathrm{e}}$ siècle à 830000 stères vers 1720 , puis à environ 1,5 million à la fin de l'Ancien Régime ${ }^{14}$. Cela équivaut à près de 4000 stères, soit 2000 à 3000 tonnes par jour, qui transitent par les deux ports aux bois du cœur de Paris, le port de Grève pour les bois venant d'amont et celui de l'École pour ceux d'aval. La satisfaction de cette demande jusqu'aux années 1770 tient même du miracle. Elle s'explique par une importante extension des aires d'approvisionnement, par un drainage systématique et poussé des forêts du Bassin parisien, rendu possible par le flottage des bois. À la croissance démographique peut-on enfin ajouter une dernière cause, sans doute importante, mais qu'il conviendrait encore de mesurer avec précision: la demande en bois de marine. Durant le ministériat de Choiseul, la France s'est dotée d'une politique navale d'envergure, assez comparable à celle menée un siècle plus tôt par Colbert, et générant des difficultés d'approvisionnement relativement similaires. Un navire de ligne consomme environ 3000 chênes centenaires, 6000 à $10000 \mathrm{~m}^{3}$ de bois sur pied, volumes que la forêt française peine désormais à fournir avec qualité et régularité.

\section{UNE CRISE ATTENDUE PAR LA PLUPART DES THÉORICIENS}

En réalité, cette crise est loin d'être une surprise. Depuis de nombreuses décennies, de nombreux théoriciens avaient déjà tenté de tirer la sonnette d'alarme pour montrer que la ressource était déjà en danger. Au début du siècle, le maréchal de Vauban avait sans doute été le premier à contester l'œuvre réformatrice du grand Colbert, en montrant que les normes imposées par la législation conduisaient à une impasse. Selon lui, la conversion massive d'anciennes futaies en taillis sous futaie entraînait déjà à une pénurie en bois de construction:

Il y a longtemps qu'on se plaint que les futaies se ruinent, qu'elles s'anéantissent partout, que dans peu elles seront réduites en taillis, et qu'incessamment nous manqueront de bois à bâtir: l'expérience de ceux qui font travailler chez eux ne vérifie que trop la justice de cette plainte, par

14 Jean BoISsièRE, La consommation parisienne de bois et les sidérurgies périphériques: essai de mise en parallèle (milieu $\mathrm{XV}^{\mathrm{e}}$-milieu $\mathrm{XIX}^{\mathrm{e}}$ siècle), dans: WORONOFF (dir.), Forges et forêts (voir n. 11), p. 35. 
la difficulté où ils sont de trouver des bois, et pour peu qu'on veuille se donner la peine d'examiner de près l'état des forêts, tant du roi que des particuliers, on s'apercevra bientôt du désordre où elles sont. On verra que toutes les futaies qui se sont trouvées de quelque débit, ont été coupées; que les particuliers se sont défaits de tout ce qu'ils avaient de meilleur à cet égard; ce qui est parvenu à tel excès qu'on ne trouve plus de bois à bâtir qu'avec beaucoup de peine et en l'achetant bien cher dans les lieux mêmes qui en étaient couverts il n'y a pas soixante ans. On verra que ce mal s'accroît tous les jours de plus en plus par la coupe continuelle du peu qu'il en reste sur pied; en sorte que si bientôt on n'y remédie, on sera obligé de chercher du bois à bâtir hors du royaume ${ }^{15}$.

L'argument le plus pertinent s'appuyait sur l'analyse comparée des revenus du taillis et de la futaie, qui montrait qu'une futaie produit toujours davantage que le taillis à temps égal, en fournissant à la fois des bois d'œuvre et des bois de feu. Elle est confirmée et prolongée en 1739 par Réaumur et Buffon, dans les »Mémoires de l'Académie des Sciences ${ }^{16}$. Mais la première analyse très fouillée des lacunes du modèle colbertien revient à Duhamel du Monceau, dans son »Traité général des forêts« (1755-1767). Inspecteur général de la Marine et chargé à ce titre de l'approvisionnement des chantiers navals en bois de marine, il est l'un des plus grands connaisseurs de l'époque en matière de sylviculture. Selon lui, la crise s'est aggravée, touchant désormais tous les secteurs du royaume:

Les bois sont presque d'une première nécessité, soit pour nous défendre des rigueurs du froid, soit pour la préparation de nos aliments, pour l'exploitation des mines, pour le soutien de quantité de manufactures, telles que les verreries, savonneries, brasseries, tuilleries, faïenceries; les ateliers des teinturiers, les fours à chaux, pour l'établissement de quantité de machines, écluses, moulins, usines; pour les charpentes des bâtiments civils [et] militaires, pour la navigation sur les rivières; enfin pour la marine, qui en fait indispensablement une prodigieuse consommation [...]. La disette de cette matière se fait déjà sentir de toute part: le pauvre ne peut qu'à gros frais se garantir de la rigueur de nos hyvers; les manufactures sont forcées de porter à un prix fort haut leurs marchandises, ou d'interrompre leurs fabriques, les propriétaires des maisons dans les villes, ou de fermes à la campagne, s'aperçoivent que l'entretien des charpentes de leurs bâtiments devient tellement onéreux, qu'une réparation, en apparence médiocre, consomme les loyers, nonseulement d'un mois mais même de plusieurs années. Enfin tous ceux qui emploient le bois en œuvre, éprouvent que cette matière est portée à un prix excessif ${ }^{17}$.

Après Duhamel, tous les théoriciens font le constat d'une crise d'approvisionnement, marquée par une hausse des prix des bois, une pénurie de combustible comme de bois d'œuvre. Parmi les plus connus peut-on citer le forestier Pannelier d'Annel, artisan de

15 Sébastien DE VAuban, Traité de la culture des forêts, 1701, dans: ID., Oisivetés de M. de Vauban, t. II, Paris 1843, p. 59-60.

16 Michel DeVEZE, La crise forestière française dans la $1^{\text {ère }}$ moitié du XVIII ${ }^{\mathrm{e}}$ siècle et les suggestions de Vauban, Réaumur, Buffon, dans: Actes du $88^{\mathrm{e}}$ congrès national des Sociétés savantes, Clermont-Ferrand 1963. Section d'histoire moderne et contemporaine, Paris 1964, p. 595-616, rééd. dans: ID., La forêt et les communautés rurales, $\mathrm{XVI}^{\mathrm{e}}-\mathrm{XVIII}{ }^{\mathrm{e}}$ siècles, Paris 1982, p. 63-84.

17 Duhamel du Monceau, Des semis et plantation des arbres et de leur culture, Paris 1760, p. IV-V; cf. Jérôme BuRIDANT, Duhamel du Monceau et la crise forestière du XVIII ${ }^{\mathrm{e}}$ siècle, dans: Andrée CoRvol (dir.), Duhamel du Monceau, 1700-2000: un Européen du siècle des Lumières, Orléans 2001, p. 41-54. 
la restauration de la forêt de Compiègne, l'ingénieur du duc d'Orléans Plinguet, le grand maître des Eaux et Forêts de Champagne Tellès d'Acosta, le louvetier Delisle de Moncel, l'agronome Varenne de Fenille ou le correspondant de la Société d'Agriculture Baillon ${ }^{18}$. S'ils constatent généralement que la pénurie peut être renforcée par la croissance des besoins en bois, tous s'accordent pour chercher en forêt les racines du mal en parlant d'un »dépérissement des bois« ou d'un »dépérissement forestier«.

Derrière ces termes se cache une crise environnementale assez sensible, qui se manifeste de nombreuses manières. Une des premières manifestations de cette crise semble être une évolution régressive des peuplements forestiers. Alors qu'une succession progressive de la végétation, en l'absence de perturbation majeure, aboutirait selon les sols à la chênaie-hêtraie ou à la hêtraie pure, on enregistre dans de nombreux massifs des faciès régressifs assez marqués, qui aboutissent dans le meilleur des cas à la chênaie-charmaie, la chênaie-frênaie ou la bétulaie, parfois même à des prés-bois et à des landes dégradées ${ }^{19}$. Cette dynamique, décrite avec précision dans les procès-verbaux de visite de bois, reste encore mal expliquée par les forestiers. En s'inspirant du principe de la rotation des cultures sur les terres agricoles, on imagine encore que la terre n'accepte pas la succession des mêmes productions, une production uniforme causant à terme un épuisement du sol. En réalité, l'épuisement des sols forestiers peut seulement apparaître lors de coupes très rapprochées, avec des rotations courtes inférieures à quinze ans. Cette situation est fréquente dans les bois particuliers comme dans les bois communaux. Elle est devenue assez rare dans les bois royaux et ecclésiastiques, où les rotations ont eu tendance à être augmentées au fil du siècle. Dans ces circonstances, la dynamique régressive des peuplements peut surtout s'expliquer par la mauvaise mâ̂trise des régénérations: coupes trop fortes, insuffisance et mauvaise qualité des réserves, irrégularité des fructifications, etc.

Une seconde manifestation de cette crise forestière est la sensibilité aux chablis. Comme aujourd'hui, le terme de »chablis« désigne déjà, à l'époque moderne, les arbres déracinés ou cassés par les éléments naturels comme le vent ou la neige. Au sens plus étroit, les forestiers distinguent plus généralement les chablis effectivement déracinés ou »culevés« et les »volis« cassés, la »chandelle« restant encore sur pied.

18 PAnNelier D'AnNel, Essai sur l'aménagement des forêts, présenté au roi, Paris 1778; J. B. PLINGUET, Traité sur les réformations et les aménagements des forêts avec une application à celles d'Orléans et de Montargis, Orléans 1769; ID., Examen analytique des causes du dépérissement des bois, $2^{\mathrm{e}}$ éd., Orléans, s.d.; TELLÈs D’ACOSTA, Instruction sur les bois de marine, contenant des détails relatifs à la physique et à l'analyse du chêne, et en ce qui concerne l'économie et l'amélioration du bois en général, Paris 1780; Delisle DE MonCEL, Mémoire sur le repeuplement, l'augmentation et la conservation à venir des bois dans les départements de la Meurthe, Moselle, Aisne, Meuse, Marne, etc., Nancy 1791; P. C. VARENNE DE FEnILLE, Mémoires sur l'administration forestière et sur les qualités individuelles des bois indigènes ou qui sont acclimatés en France, Bourg ${ }^{2}$ 1792; Philibert LE DuC (dir.), CEuvres agronomiques et forestières de Varenne de Fenille, Paris 1869; J. F. E. BAILlon, Causes du dépérissement des bois. Quelles sont les causes du dépérissement des bois? Quels sont les moyens d'y remédier?, Paris 1791.

19 Par exemple à Saint-Gobain, Compiègne, Halatte, Fontainebleau, Chantilly, dans une moindre mesure à Clairvaux, en Argonne. 
Les épisodes de chablis sont désormais bien connus par un programme de recherches mené par le Groupe d'histoire des forêts françaises ${ }^{20}$. Du XVI ${ }^{\mathrm{e}}$ au XX $\mathrm{XX}^{\mathrm{e}}$ siècle, le temps de retour au vent de plus de $120 \mathrm{~km} / \mathrm{h}$, seuil significatif pour produire des dégâts en forêt, apparaît identique à celui des trente dernières années, de l'ordre de cinq à six ans. Cet ordre de grandeur cache, bien entendu, des irrégularités chronologiques, certaines périodes apparaissant plus agitées que d'autres, comme la fin du XVI ${ }^{\mathrm{e}}$ siècle, le $\mathrm{XVIII}^{\mathrm{e}}$ siècle, la fin du $\mathrm{XIX}^{\mathrm{e}}$ ou du $\mathrm{XX}^{\mathrm{e}}$ siècle. La recrudescence des chablis éoliens dans le courant du XVIII ${ }^{\mathrm{e}}$ siècle pose des problèmes d'interprétation. Les documents forestiers font en effet apparaître une sensibilité accrue aux grands vents, avec une accélération sensible de la récurrence des épisodes de casse, notamment en 1716, 1717, $1726,1729,1735,1736,1739,1741,1746,1751,1756,1765,1781$ ou 1786. Si les martelages de chablis se font de plus en plus nombreux durant cette période, les mémoires et les journaux intimes livrent très peu de descriptions de tempêtes, contrairement au siècle précédent. Cette recrudescence de chablis, souvent provoqués par des vents d'assez faible intensité, peut s'expliquer par une fragilisation des peuplements.

\section{Arbres déclassés en forêt de Saint-Gobain, 1650-1760}

(moyenne mobile sur trois ans)

Source: Arch. dép. Aisne B 3563-3598

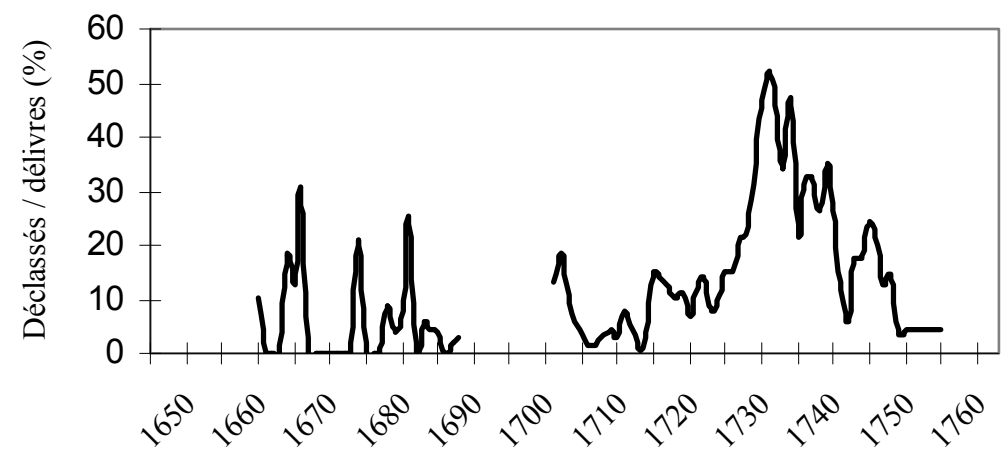

Cette fragilisation est par exemple évidente en forêt de Saint-Gobain, où l'on observe une hausse impressionnante des arbres déclassés lors des martelages en abandon. Ces arbres, dénommés »hallots«, sont souvent »morts«, »morts en cime«, »pourris« ou tarés, peut-être attaqués par les insectes. Ce phénomène de dépérissement est aussi re-

20 Andrée Corvol (dir.), Tempêtes sur la forêt française, $\mathrm{XVI}^{\mathrm{e}}-\mathrm{XX}^{\mathrm{e}}$ siècle, Paris 2005; Jérôme BURIDANT, L'impact des variations climatiques sur les forêts de plaine du nord-est de la France, entre le $\mathrm{XVI}^{\mathrm{e}}$ et le XIX ${ }^{\mathrm{e}}$ siècle, dans: Jean-Pierre CHABIN (dir.), La forêt dans tous ses états: de la Préhistoire à nos jours, Besançon 2005, p. 57-72. 
marqué au même moment dans la maîtrise des Eaux et Forêts de Nancy ${ }^{21}$. Sans que cela soit explicite dans les documents de l'époque, on peut légitimement s'interroger sur les rapports entre ce dépérissement, net et brutal, et les effets du »grand hyver « de 1709. Après 1709 , les forestiers ont nécessairement purgé les coupes des arbres morts, mais ont le plus souvent tenté de préserver l'avenir en maintenant le maximum de semenciers, même entre vie et mort. Une révolution plus tard, le destin de ces arbres de réserve apparaît évident. Morts en cime, viciés par les insectes, ils n'ont même plus assez de valeur pour être vendus au cours ordinaire, et tombent à chaque grand vent d'hiver. Ce phénomène de dépérissement peut être parfois corroboré par des mentions, plus rares, de dégâts de »chenilles«. Toutes ces indications sont révélatrices de déséquilibres environnementaux profonds, autrement dit d'une perturbation sensible des écosystèmes forestiers, qui n'est pas sans conséquence sur leur productivité. La crise énergétique semble bien parallèle à une grave crise environnementale.

\section{Prix des bois flottés rendus à Paris au $\mathrm{XIX}^{\mathrm{e}}$ siècle}

Sources: Annales des Eaux et Forêts, Revue forestière française

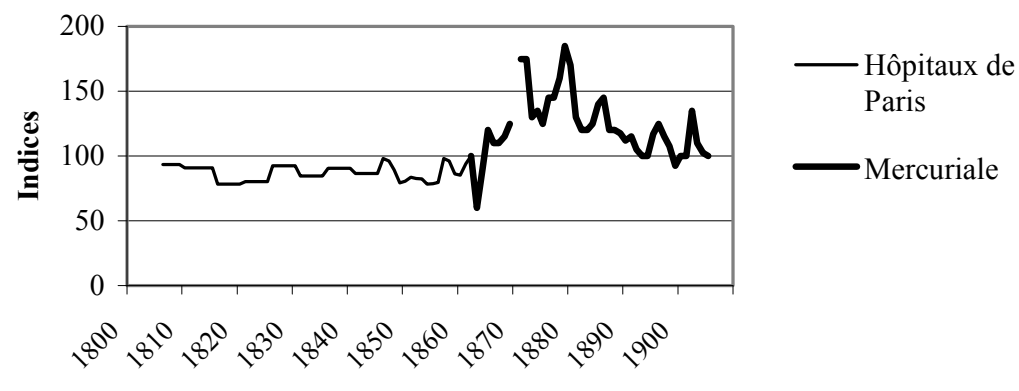

\section{LE PARADOXE FORESTIER: UNE CRISE RETARDÉE}

En dépit de l'ampleur de la menace, on constate au XIX ${ }^{\mathrm{e}}$ siècle des évolutions qui paraissent totalement paradoxales. Alors que les industriels britanniques passent majoritairement au charbon de terre, les Français continuent majoritairement la chauffe au bois très en avant au cours du siècle. Dans notre pays, l'apogée de la sidérurgie au bois se place même en 1856. À cette date, le premier département sidérurgique français est encore la Haute-Marne, et le second la Haute-Saône, témoins d'une géographie industrielle totalement différente de celle du $\mathrm{XX}^{\mathrm{e}}$ siècle, encore calquée sur la carte des disponibilités forestières. Cette résistance s'explique principalement par un second paradoxe, celui des prix. De 1810 à 1860, les prix des bois enregistrent une relative stabilité, qui contredit l'imminence de la crise.

21 Travaux de Daniel BERNI, présentés de manière synthétique par Christian DugAS DE LA BoISSONNY, Repérer le dépérissement, dans: Andrée CORVOL (dir.), La forêt malade: débats anciens et phénomènes nouveaux, Paris 1994, p. 69-88. 


\section{DES STRATÉGIES INDUSTRIELLES DIFFÉRENTES}

Dans le détail, on s'aperçoit que la plupart des industries au bois retardent au maximum le passage aux chauffes au charbon de terre, et ce pour de multiples raisons. Le cas de la Manufacture royale de grandes glaces de Saint-Gobain peut, à cet égard, fournir un bon exemple. Implantée à Saint-Gobain depuis 1692, la glacerie connaît un essor particulièrement considérable à partir du directorat de Pierre Delaunay-Deslandes (1758). Celui-ci entreprend une restructuration totale du site, réorganisant les fours, supprimant le soufflage, améliorant inlassablement les techniques de coulage. Avec une croissance continue, à peine interrompue par la Révolution, la Manufacture devient en 1845 la quatrième entreprise française. En dépit de la proximité de la forêt, la glacerie est confrontée à un problème structurel d'approvisionnement en bois. Dans les années 1770, elle consomme bon an mal an 30000 stères de bois, son bassin d'approvisionnement couvrant un massif de plus de 10000 ha. À cette date, l'envolée des prix commence à inquiéter, alors qu'ils n'avaient pas représenté un obstacle considérable à la production durant des décennies. Deslandes, qui envisage de passer à des chauffes au charbon de terre, entreprend alors de multiples essais techniques. La mutation énergétique ne lui paraît pas impossible puisqu'elle connaît des précédents. Le charbon de terre est déjà utilisé depuis 1605-1610 en Angleterre, et depuis 1749 en Normandie, à la verrerie de Tourlaville, le charbon étant importé d'outre-Manche. Il s'agit néanmoins d'établissements qui produisent du verre soufflé, avec des techniques différentes. Les premiers essais gobanais sont loin d'être concluants. Après la construction d'un four à charbon, on se rend compte que des escarbilles sont projetés dans les creusets, et que la fumée grasse colore le verre en noir. Ces difficultés ne sont certes pas insurmontables, mais elles se doublent d'obstacles d'ordre économique. En l'absence de mines à proximité de la glacerie, le charbon provient des mines d'Anzin (Nord), ouvertes depuis 1734. Au départ d'Anzin, le charbon de terre reste moins cher que le bois, mais le transport par terre et partie par voie d'eau, sur plus d'une centaine de kilomètres, multiplie par quatre le prix initial. Dans ces conditions, la chauffe au bois reste encore rentable pour plusieurs décennies, même dans des conditions de hausse des prix. À l'échelle du massif forestier, mieux vaut encore surenchérir pour emporter les ventes, étouffer la concurrence et à plus long terme arriver à une situation de monopole.

Plus que les aspects techniques, le facteur essentiel qui fait opter, soit pour le bois, soit pour le charbon, reste le rapport prix-distances. C'est ce facteur, plus que tout autre, qui explique la plupart des stratégies énergétiques industrielles. La Manufacture maintien cette stratégie jusque 1840 , date à laquelle la construction des canaux et les économies d'échelle réalisées dans les mines rendront davantage rentable l'approvisionnement en charbon. Jusqu'à ce moment s'appliquent surtout des logiques d'économie: amélioration du rendement des fours, meilleure surveillance du personnel... En forêt, la pression économique pèse aussi fortement sur les aménagements forestiers, entraînant progressivement une normalisation de la production. En 1769, la forêt de Saint-Gobain passe de la famille Mazarin à l'apanage d'Orléans. Dès 1770, la Maison d'Orléans impose un nouvel aménagement, qui modifie l'ensemble du parcellaire et 
convertit l'ensemble des peuplements au taillis sous futaie à la révolution de 25 ans, traitement parfaitement adapté à la demande verrière ${ }^{22}$.

Comme on l'a dit, la substitution énergétique est encore plus tardive dans la sidérurgie. En France, la prééminence des maîtres de forge haut-marnais dure jusqu'aux années 1870-1873. Ils pratiquent une fonte au bois, selon la technique du haut fourneau mise au point dans la région de Liège au $\mathrm{XIV}^{\mathrm{e}}$ siècle. Leur déclin ne sera véritablement consommé qu'avec la Grande dépression (1873-1896), qui jouera surtout en faveur des sidérurgies modernes, plus rentables. Il sera aussi renforcé par l'essor de la sidérurgie lorraine voisine après 1876, date de l'invention du procédé de SidneyGilchrist Thomas, permettant de décarburer les fontes phosphoreuses. Le plus paradoxal encore reste le développement des bas fourneaux et des forges dites à la Catalane. La technique du bas fourneau, déjà au point dans l'Antiquité, demeure assez rudimentaire et produit des fontes de qualité inégale selon le minerai. Elle connaît cependant un essor assez remarquable dans les Pyrénées de l'est (Ariège, Aude, Pyrénées orientales) et le Dauphiné, le maximum de production se situant en... 1852! Si la technique reste archaïque, elle demande peu de savoir-faire, une masse de capitaux encore limitée, et reste surtout très économe en combustible, contrairement aux hauts fourneaux. Ici encore, c'est le prix du combustible qui détermine les choix industriels $^{23}$. Si de nouvelles géographies industrielles apparaissent progressivement (Nord, près d'Anzin, ou Loire, au Creusot), les vieilles industries s'appuient encore longtemps sur leurs anciennes logiques, la rupture la plus importante étant l'arrivée du chemin de fer, qui bouleverse brutalement les possibilités d'échange.

Ces données fondamentales sont renforcées par des facteurs internes. Avec la vente des biens nationaux durant l'épisode révolutionnaire, et plus encore avec les ventes massives de bois durant la Restauration, les industriels ont pu progressivement se porter acquéreurs d'espaces boisés, pour s'affranchir du marché. Comme dans les verreries, on observe aussi parallèlement des logiques d'économie, avec une amélioration des fours (meilleur tirage, récupération de la chaleur), une amélioration considérable des rendements, qui permet au bois d'être rentable durant plus d'un demi-siècle. Des logiques similaires d'économie et d'amélioration des rendements sont aussi remarquées pour le chauffage domestique, produisant les mêmes effets ${ }^{24}$.

22 BURIDANT, Espaces forestiers et industrie verrière (voir n. 8), p. 188-206, 217-230.

23 Jérôme BONHÔTE, Christian FruHAUf, La métallurgie au bois et les espaces forestiers dans les Pyrénées de l'Aude et de l'Ariège, dans: WorOnOFF (dir.), Forges et forêts (voir n. 11), p. 151-212.

24 Jean-François BeLhoste, Le chauffage à Paris: approvisionnement en bois et évolution des appareils $\left(\mathrm{XVII}^{\mathrm{e}}-\mathrm{XIX}^{\mathrm{e}}\right.$ siècle), dans: Le bois, source d'énergie: naguère et aujourd'hui, Paris 2000 (Cahier d'études forêt, environnement et société, $\mathrm{XVI}^{\mathrm{e}}-\mathrm{XX}^{\mathrm{e}}$ siècle, 10), p. 58-61. 


\section{UNE RÉPONSE FORESTIĖRE?}

Si la crise peut être en partie contenue par la modération de la demande, qu'en est-il de l'évolution de l'offre? En d'autres termes, la stagnation des prix dans la première moitié du $\mathrm{XIX}^{\mathrm{e}}$ siècle peut-elle s'expliquer par une augmentation de la production sylvicole?

Le XIX ${ }^{e}$ siècle est d'abord marqué par une réorganisation complète du corps forestier, qui passe progressivement d'une structure judiciaire à une structure technique. La période révolutionnaire se caractérise par une grande période d'incertitudes et de bouleversements internes, le moment le plus marquant restant le rattachement de l'administration des Eaux et Forêts à la Régie de l'enregistrement et des domaines (4 brumaire an IV). De 1796 à 1877, le corps forestier reste intégré à l'administration des Finances, les logiques économiques s'imposant de fait ${ }^{25}$. C'est surtout durant l'Empire que s'impose l'influence allemande. Avec l'annexion de la Rhénanie à la France, et la création des départements de la Roer, de la Sarre, de Rhin et Moselle et de Mont-Tonnerre, l'administration forestière tente d'imposer ses institutions et ses lois Outre-Rhin. Mais les forestiers français envoyés sur place découvrent en retour toute l'avance de la sylviculture allemande. Parmi ceux-ci émerge la figure de JacquesJoseph Baudrillart, premier traducteur en Français des grands théoriciens allemands comme Georg-Ludwig Hartig (»Anweisung zur Holzzucht«, 1791), et vulgarisateurs des méthodes allemandes (régénérations par coupes progressives, méthodes de conversion...). Devenu chef de division à l'Administration générale des Eaux et Forêts, il est l'un des artisans de la création de l'École royale des Eaux et Forêts de Nancy, en 1824. Contrairement au corps des Ponts et Chaussées ou à celui des Mines, le corps forestier ne s'était pas encore doté d'une école formant des ingénieurs aux sciences modernes. Le contraste avec l'Allemagne est particulièrement marquant, car dans de nombreux États se sont déjà ouvertes des écoles, à Stuttgart dès 1772, à Göttingen en 1775 , à Zillbach en Saxe en 1785, à Munich en 178726. Le choix de Nancy n'est d'ailleurs pas fortuit. La proximité de l'Allemagne permet des échanges fructueux, et les trois premiers directeurs, Lorentz, Salomon et Parade, sont Alsaciens et ont fait leurs études en Allemagne. Le nouveau code forestier, promulgué en 1827, reste cependant encore très hésitant quant à la démarche à suivre. Héritier des principes de la Révolution, il reste très libéral pour la forêt privée, plus directif pour les forêts domaniales. Sur le terrain, les aménagements restent pourtant tardifs. Ils se multiplient entre 1850 et 1870 , au moment où les prix commencent à s'emballer.

La politique de plantation, qui commence timidement au XVIII ${ }^{\mathrm{e}}$ siècle, s'accélère surtout sous la Monarchie de Juillet et le Second Empire. C'est le moment du boisement des Landes de Gascogne en pin maritime, de la Champagne en pin sylvestre puis en pin noir d'Autriche, de la Sologne et des dunes littorales de la Manche ${ }^{27}$. Ces plan-

25 De 1877 à 1964, les Eaux et Forêt sont rattachées au ministère de l'Agriculture.

26 Michel DEVÈzE, Forêts françaises et forêts allemandes: étude historique comparée, dans: Revue historique 480 (1966), p. 57-67.

27 Jean-Robert PITTE, Histoire du paysage français, t. II: Le profane: du $16^{\mathrm{e}}$ siècle à nos jours, Paris 1983, p. 86-88; Louis BADRÉ, Histoire de la forêt française, Paris 1983, p. 131-176. 
tations, désormais massives, ne portent cependant leurs fruits qu'au terme du premier cycle de production. Les premières coupes interviennent réellement après 1870 , au moment où les prix des bois commencent à s'effondrer, la substitution énergétique étant désormais bien engagée.

L'apaisement des tensions sur le marché, dans la première moitié du XIX ${ }^{\mathrm{e}}$ siècle, ne provient donc ni d'une amélioration rapide de la gestion forestière, ni d'une augmentation des surfaces. S'il faut chercher une origine interne à la forêt, il faut seulement évoquer la possibilité d'une décapitalisation progressive des réserves accumulées au XVIII ${ }^{\mathrm{e}}$ siècle, phénomène qu'il conviendrait de mesurer avec plus de finesse à l'échelle des massifs.

La réalité de la crise forestière, entre 1770 et 1860 reste l'objet de débats. La croissance industrielle, alliée à la croissance démographique et urbaine, entraine bien une hausse des besoins. À bien y regarder, la forte hausse des prix, spectaculaire pour les contemporains, n'est peut-être pas un phénomène si anormal, après des siècles où la ressource ligneuse s'était débitée à vil prix. Sous cet angle, l'envolée vertigineuse des prix dans la période 1770-1800 peut-être aussi vue comme une »reprise technique«, un réajustement de l'offre à la demande. La hausse, il est vrai, s'explique aussi par une insuffisance de la production, sans doute liée à une crise environnementale assez sensible. Hormis quelques exceptions, les peuplements forestiers apparaissent généralement très dégradés, avec de forts déséquilibres environnementaux, mais aussi de forts contrastes dans la gestion, avec des secteurs réellement surexploités et d'autres encore totalement laissés à l'abandon.

En dépit des alertes et des prophéties de malheur, la pénurie n'apparaît cependant pas réellement avant le milieu du XIX ${ }^{\mathrm{e}}$ siècle. Cette crise retardée appelle de nombreuses hypothèses d'explication. Les logiques de rationalisation de la ressource et de son emploi, les logiques d'économie, l'amélioration des réseaux de transport et la meilleure monopolisation des potentialités peuvent en grande partie expliquer la modération des prix au début du $\mathrm{XIX}^{\mathrm{e}}$ siècle. On peut aussi remarquer que l'alerte semblait certainement aussi disproportionnée. Comme toujours dans l'histoire, l'angoisse de l'avenir l'emporte toujours sur la réalité des faits.

\section{DEUTSCHE ZUSAMMENFASSUNG}

Während der gesamten Frühen Neuzeit (16.-18. Jahrhundert) gab es in Frankreich wiederholt Holzmangelkrisen (1661-1662, 1714-1715, 1740-1742), die die Zuspitzung der Situation mit einem starken Preisanstieg in der Zeit zwischen 1770 und 1800 ankündigten. Seit Beginn des 18. Jahrhunderts hatten die Forstwissenschaftler diese Krise vorhergesagt und vor allem nach den internen Ursachen für den Holzmangel gesucht. Die Holznot des späten 18. Jahrhunderts war teilweise auf eine ernste Umweltkrise zurückzuführen, die die Holzproduktion belastete, aber auch Folge des ansteigenden Konsums in den Städten und in der Industrie. Trotz aller Alarmrufe zogen die Industriellen lange Zeit die Holzfeuerung anderen Arten der Energiegewinnung vor. Forciert wurde dieses Verhalten durch technische Fortschritte, die Einsparungen beim Brennmaterial erlaubten, und insbesondere durch den Preisrückgang für Holz in der ersten Hälfte des 19. Jahrhunderts. Dieser war in erster Linie eine Folge der stärkeren Ausschöpfung der Ressourcen bzw. der Vermarktung der Reserven, weniger jedoch ein Ergebnis rationeller Bewirtschaftung der Forste oder einer Ausweitung der forstwirtschaftlich genutzten Flächen. 